PRION DISEASE

\title{
'Anti-prions' block prion disease onset
}

Treatment with an artificial, nontoxic prion protein $(\mathrm{PrP})$ could substantially delay or even prevent the development of prion disease, according to a new study in a hamster model of prion disease. Researchers found that synthetic prions - dubbed 'antiprions' - competed with pathogenic $\mathrm{PrP}$ to protect the animals against the disease.

Prion diseases are fatal neurological conditions for which no treatments currently exist. These disorders are caused by misfolded PrP, which induces normally folded cellular PrP to undergo the same aberrant conformational change. This process results in accumulation and aggregation of the pathogenic protein form in the brain, and leads to the development of disease.

Previous work suggested that different strains of prions compete with each other and cause distinct disease characteristics. Claudio Soto

similar agents could be produced to treat other more common neurodegenerative disorders such as Alzheimer and Parkinson disease and colleagues investigated whether this competition could be exploited as a novel way to treat the disease. "Our strategy was to produce a new form of abnormal PrP that retains the ability to convert the normal PrP, but creates a product that is not toxic and does not cause disease," explains Soto. "In this manner, this alternative form - which we call an anti-prion - converts the normal protein into an innocuous form so that normal protein is not available to be converted into the pathological form during prion infection."

The team purified PrP from bacteria that were transfected with a plasmid containing the PrP gene, then denatured and annealed the protein to form anti-prion aggregates. They injected the anti-prions into hamsters that were infected with the pathogenic $263 \mathrm{~K}$ strain of PrP. Animals treated with anti-prions at the same time as or prior to infection with $263 \mathrm{~K}$ prions survived longer than control $263 \mathrm{~K}$-infected animals. The anti-prions also completely prevented disease in a proportion of hamsters that were treated with low concentrations of $263 \mathrm{~K}$ prions.

The researchers then used immunostaining to examine the distribution of $\operatorname{PrP}$ in the brains of infected hamsters. Animals treated with the anti-prion had an altered distribution of PrP staining: $\operatorname{PrP}$ deposition in certain brain areas of anti-prion-treated animals was considerably less than that in control 263K-infected animals. Furthermore, the team found that the anti-prions could inhibit conversion of normal PrP to pathogenic PrP by $263 \mathrm{~K}$ prions in vitro, supporting the theory that the anti-prions exert their effect by interfering with PrP replication.

Soto and colleagues now plan to develop more-potent anti-prions that might present an option for future clinical trials in human prion disease. The team are also investigating whether similar agents could be produced to treat other more common neurodegenerative disorders, such as Alzheimer and Parkinson disease, as evidence suggests that aggregation of the proteins involved in these diseases can propagate and be transmitted in a similar way to prions.

Charlotte Ridler

ORIGINAL ARTICLE Diaz-Espinoza, R. et al. Treatment with a non-toxic, self-replicating anti-prion delays or prevents prion disease in vivo. Mol.Psych. http://dx.doi.org/10.1038/mp.2017.84 (2017) 